\title{
Identifying eating habits in Finnish children: a cross-sectional study
}

\author{
Rejane Augusta de Oliveira Figueiredo ${ }^{1,2^{*}}$ (D), Jannina Viljakainen ${ }^{1,2}$, Heli Viljakainen ${ }^{1,3}$, Eva Roos ${ }^{1,4}$, \\ Trine B. Rounge ${ }^{1,2,5 \dagger}$ and Elisabete Weiderpass ${ }^{1,2,5,6,7 \dagger}$
}

\begin{abstract}
Background: We aimed to identify different eating habits among Finnish children and to evaluate their association with meal patterns, breakfast consumption, and socio-demographic characteristics in a large, nationwide cohort of children.

Methods: We evaluated 10,569 children aged 9-14 years into the Finnish Health in Teens cohort in a cross-sectional design. The hierarchical K-means method was used to identify groups of children with different eating habits, based on five factors obtained through factor analysis of 10 food items. Multiple correspondence analysis was used to show associations between groups with different eating habits and meal patterns, breakfast patterns, gender, age, and language spoken at home.

Results: Analyses identified three groups: unhealthy eaters (12.3\%), fruit and vegetable avoiders (43.3\%), and healthy eaters (44.1\%). Most children had regular meal and breakfast patterns. The proportion of boys was higher among unhealthy eaters. Unhealthy eaters also showed irregular meal and breakfast patterns, and had parents with low education level. There was a higher proportion of girls among healthy eaters. Healthy eaters also showed regular meal and breakfast patterns, and had parents with high education level.

Conclusions: Although the number of unhealthy eaters was small, special attention should be still paid to these, mostly male children, as they have poor eating habits and they lack regular eating routine. Skipping breakfast was more common among older children and girls, although girls had healthier eating habits overall. Our results can contribute to public health efforts to improve eating behaviours, especially among children with poor eating habits and those skipping healthy food items.
\end{abstract}

Keywords: Eating habits, Healthy eating, Breakfast, Meal pattern, Children, Finland, Epidemiology

\section{Background}

At present, countries worldwide are focusing on fostering a healthy diet and healthy eating habits, which are major determinants of health and disease [1], including the development of overweight and obesity [2]. Due to the pandemic of childhood obesity [3], the eating habits of children and adolescents are of particular importance, as unhealthy eating habits in childhood/adolescence can persist and cause adverse health outcomes in adulthood $[4,5]$.

\footnotetext{
* Correspondence: rejane.figueiredo@helsinki.fi

${ }^{\dagger}$ Trine B. Rounge and Elisabete Weiderpass contributed equally to this work.

${ }^{1}$ Folkhälsan Research Center, Biomedicum 1 Helsinki, PB 63 (Haartmansgatan 8), 00014 University of Helsinki, Helsinki, Finland

${ }^{2}$ Faculty of Medicine, University of Helsinki, Helsinki, Finland

Full list of author information is available at the end of the article
}

A study carried out in 124 developed and developing countries showed an improvement in worldwide dietary quality from 1980 to 2009 , with an increased availability of energy from vegetable oils, fruits, and vegetables, and a decreased availability of energy from sugar and animal fats [6]. Over the last years, the Finnish diet among working-age adults has also improved, with an increase in the consumption of fruits and vegetables and a decrease in the consumption of sweets and soft drinks $[7,8]$.

However, Hoppu et al. [9] reported that the main dietary concerns among Finnish adolescents are low consumption of fruits and vegetables and high consumption of sucrose-rich drinks and snacks. Other studies have reported that young children commonly consume skimmed milk, low-fat cheese or cold-cuts, and

(c) The Author(s). 2019 Open Access This article is distributed under the terms of the Creative Commons Attribution 4.0 International License (http://creativecommons.org/licenses/by/4.0/), which permits unrestricted use, distribution, and reproduction in any medium, provided you give appropriate credit to the original author(s) and the source, provide a link to the Creative Commons license, and indicate if changes were made. The Creative Commons Public Domain Dedication waiver (http://creativecommons.org/publicdomain/zero/1.0/) applies to the data made available in this article, unless otherwise stated. 
vegetable oil-based margarine on bread, but rarely fish $[10,11]$. It was estimated that beverages and foods consumed between meals provide as much as $42 \%$ of total daily energy intake, and the quality of these food items is of concern [11]. Similar results have been reported in young male conscripts in Finland [12], among whom daily consumption of fruits, berries, and vegetables was rare, and consumption of rye bread, dairy products, and sugar-sweetened soft drinks was favoured. On average, the food consumption of these young men fulfilled less than half of the Nordic Nutrition Recommendations [12].

Healthy eating also includes a consistent meal pattern, and such patterns have been the focus of several studies $[13,14]$. The conventional daily Finnish meal pattern includes breakfast, a warm lunch, a warm dinner, and two snacks [15]. Breakfast is consumed daily by $61 \%$ of adolescents in Finland, a number which has remained stable between 2002 and 2010 [16], and by $87 \%$ of primary school pupils [13]. Among primary and secondary school pupils, 89 and $71 \%$, respectively, have daily school lunch, which is free of charge in public schools in Finland $[9,13]$.

As it is challenging to distinguish the effect of individual nutrients and foods on health and disease, a whole-diet approach, i.e., describing the combinations in which foods are consumed, is warranted to understand the synergistic and cumulative effects of diet on multiple health outcomes [17]. Few studies have described eating habits in the Finnish young population, and some have evaluated only the intake of specific nutrients and foods in pre-adolescents and adolescents. However, less is known about general eating habits and meal patterns in adolescence $[9,18,19]$ and the association of these factors with socio-demographic attributes. Thus we aimed to identify different eating habits among Finnish children and to evaluate the association between these eating habits and meal patterns, breakfast consumption, and socio-demographic characteristics in a large, nationwide cohort of children.

\section{Methods}

\section{Participants and cohort details}

We used data from the Finnish Health in Teens (Fin-HIT) study, a prospective cohort consisting of 11,407 pre-adolescents and adolescents (henceforth denoted as children in this study) and 10,000 parents or other adults responsible for those children (referred as parents in this study), mostly mothers. The participation rate $(30 \%)$ and details on Fin-HIT cohort were described elsewhere [20]. All children were aged 9-14 years at the time of recruitment, mostly from schools, in 2011-2014. The cohort covered a large part of Finland, including Uusimaa, Varsinais-Suomi, Häme, Pirkanmaa, Keski-Suomi, Pohjois-Savo, and Pohjois-Pohjanmaa.
In the present analysis, we included all children with baseline information on diet, consumption of selected food items, and frequency of consumption of different meals $(n=10,569)$.

\section{Information on socio-demographic characteristics}

Children completed a questionnaire covering various lifestyle and health related topics as previously described [20] . Parents completed a questionnaire which covered information on education level (anything until technical high school was categorized as low education level; anything higher was classified as high education level). Information on gender, age (in years), and language spoken at home (Finnish, Swedish, or other) was obtained from consent forms or questionnaires and confirmed by linkage with the National Population Information System at the Population Register Centre. The language spoken at home was included in the study, which in a way may reflect the participant's socio-economic status and health conditions. In general, immigrants have the worst health and socio-economic condition [21, 22]. Although Finnish-speakers and the Swedish-speakers have similar living conditions in Finland, studies showed that Swedish-speakers have better socioeconomic status and health condition [23, 24].

\section{Eating habits and meal information}

Information on eating habits in the Fin-HIT study was assessed with a 14-item food frequency questionnaire (FFQ), which covered the preceding month including both school and non-school days. Long questionnaires for children and adolescents can affect their answers, resulting in some bias, so a limited number of food items was included $[25,26]$. Selected food items covered the mandatory key indicators to evaluate healthy and unhealthy children's diet habits, as suggested by the Health Behaviour in School-Aged Children (HBSC) Study protocol [27]. Mandatory items were fruits, vegetables (fresh or cooked), sweets and sugary soft drinks. Other food items included were also important and typically used in European school studies as indicators of healthy (dark grain bread; milk or soured milk; fresh juice; and water) and unhealthy eating behaviors (pizza; hamburger or hot dog; biscuits/cookies; ice cream; chocolate or sweets; salty snacks; sugary juice drinks) $[18,28$, 29]. Children self-reported the frequency of consumption of each item on a 7-point scale ranging from 0 (not consumed) to 6 (consumed several times per day).

Information on meal patterns during school days was obtained by the question, "How often do you typically eat following meals during a school week?", followed by a list of meals: breakfast, school lunch, and dinner. Respondents reported the weekly frequency of consumption of each meal on a 6-point scale ranging from never 
to 5 days a week. Children who reported consuming lunch and dinner every school day were classified as having a regular consumption on these meals (henceforth denoted as regular meal pattern); all others were classified as having an irregular meal pattern. Breakfast consumption was studied separately since several studies have shown an association between skipping breakfast and adverse health effects [13, 30,31]. Children who reported consuming breakfast every school day were classified as having a regular breakfast pattern and the others as having an irregular breakfast pattern.

\section{Ethics}

The Fin-HIT study protocol was approved by the Coordinating Ethics Committee of the Helsinki and Uusimaa Hospital District. Informed written consent was obtained from all children and from one legally responsible adult per each child (i.e. parent or legal guardian) according to the Helsinki Declaration.

\section{Statistical analysis}

All associations between categorical variables were assessed using chi-square tests. We then identified groups of children with different eating habits. We had 14 food items, however some items were highly associated, such as cooked vegetables with fresh or grated vegetables, sugary juice with soft drink, fruits or berries with fresh juice, milk with other health items. To avoid overlapping information in the cluster analysis, we excluded food items with strong mutual associations identified by chi-square tests. Therefore, cluster analyses were performed based on 10 food items: pizza; hamburger or hot dog; biscuits/cookies; sweet pastry; ice cream; salty snacks; sugary juice drinks; dark grain bread; fruit or berries; and fresh or grated vegetables or salad. With these 10 items, we carried out a factor analysis using the principal component method for factor extraction and varimax methods for rotation. The applicability of factor analysis model was evaluated by the Kaiser-Meyer-Olklin (KMO) and Bartlett's sphericity test, considering acceptable values over 0.70 and $p<0.05$, respectively [32]. To identify groups with different eating habits we used the hierarchical K-means method, using the five factors obtained through factor analysis which represented $70 \%$ of the variability of the 10 aforementioned food items. In order to evaluate the robustness of the identified groups, we selected one sample with $60 \%$ of the total data and re-ran the cluster analysis. We repeated this process five times and compared the results with those of the original group using the Kappa analysis. All comparisons showed a $p$-value of $<0.001$ and a Kappa index greater than 0.7 , indicating high agreement.

Multiple correspondence analysis is a descriptive technique which allows researchers to visualize the relationship between several categorical variables in a graphic display [33], the closer the categories, the higher the association between them. In order to visualize different dietary behaviours among children, a multiple correspondence analysis was performed to evaluate the association between groups with different eating habits, meal patterns, breakfast patterns, gender, age, and language spoken at home. Parental education level was not included in this analysis as it was only available for 5572 children. All statistical analyses were conducted using SPSS statistical software version 24.0 and we adopted a $5 \%$ statistical significance level for all tests.

\section{Results}

There were 5564 (52.6\%) girls and 5005 (47.4\%) boys included in the analysis. Average age was $11.14( \pm 0.85)$ years, in which $61.1 \%(n=6457)$ of participants were 11 years old. Among children with information available on parental education level $(n=5572 ; 52.7 \%), 55 \%(n=3063)$ had parents with a high education level and $45 \%(n=2509)$ had parents with low education level. Regular meal pattern was observed in $75.7 \%(n=8001)$ of children, meaning that they had school lunch and dinner every school day, and regular breakfast pattern was observed in $81.0 \%(n=8563)$.

Factor analysis revealed five factors with high adaptability to the original data $(\mathrm{KMO}=0.778 ; p<0.001$ for Bartlett's sphericity test) and a high explanation of the variability of the data $(70.1 \%)$ (Table 1$)$. These factors represented five food groups: fast food (pizza; hamburger or hot dog); sweets (biscuits/cookies; sweet pastry; ice cream), salty snacks and sugary juice drinks; dark grain bread; and fruits and vegetables (fruits or berries; fresh or grated vegetables). From these factors, we obtained three groups with different eating habits: unhealthy eaters $(12.3 \% ; n=1298)$, fruit and vegetables avoiders $(43.3 \% ; n=4610)$, and healthy eaters $(44.1 \% ; n=4661)$ (factor loads can be seen in Fig. 1).

To evaluate the association between all food items and eating habits, we categorized the 7-point scale into three categories (Table 2). This revealed that unhealthy eaters consumed more food items such as pizza, hamburger or hot dog, biscuits and cookies, sweet pastry, ice cream, salty snacks, sugary juice drinks, and soft drinks. Although fruit and vegetable avoiders ate less unhealthy food items, they consumed the least fruit or berries, fresh juice, and fresh or cooked vegetables. Healthy eaters were the most frequent consumers of dark grain bread, milk, fruits or berries, fresh juice, and fresh grated or cooked vegetables, and they ate less unhealthy foods.

Unhealthy eaters showed the highest percentage of irregular meal patterns $(31.8 \% ; n=413)$, and the highest percentage of irregular breakfast patterns $(24.5 \% ; n=318)$. 
Table 1 Factor loads for each food items used in factor analysis and percentage of variance explained by each factor

\begin{tabular}{|c|c|c|c|c|c|}
\hline & \multicolumn{5}{|l|}{ Factors } \\
\hline & Sweets & Fast food & Fruit/ vegetable & Salty snacks/ sugary juice & Dark grain bread \\
\hline Pizza & 0.156 & 0.809 & -0.013 & 0.026 & 0.040 \\
\hline Hamburger or hot dog & 0.123 & 0.765 & -0.076 & 0.181 & -0.046 \\
\hline Biscuits/cookies & 0.767 & 0.094 & -0.055 & 0.237 & 0.125 \\
\hline Sweet pastry & 0.741 & 0.156 & -0.039 & 0.206 & 0.114 \\
\hline Ice cream & 0.652 & 0.326 & 0.176 & -0.095 & -0.423 \\
\hline Salty snacks & 0.219 & 0.466 & -0.041 & 0.517 & -0.037 \\
\hline Sugary juice drink (squash) & 0.212 & 0.097 & 0.091 & 0.856 & -0.068 \\
\hline Dark grain bread & 0.134 & 0.026 & 0.310 & -0.105 & 0.847 \\
\hline Fruit or berries & 0.034 & 0.003 & 0.841 & 0.048 & 0.129 \\
\hline Fresh or grated vegetables/salad & -0.054 & -0.093 & 0.842 & 0.015 & 0.101 \\
\hline Percentage of variance explained by each factor (total = 70\%) & $27.8 \%$ & $17.7 \%$ & $8.9 \%$ & $8.3 \%$ & $7.4 \%$ \\
\hline
\end{tabular}

They were also the group with a high percentage of foreign children $(4.9 \% ; n=64)$ and parents with low education level $(55.0 \% ; n=343)$ compared with other groups (Table 3). Healthy eaters had a higher percentage of regular meal patterns $(81.5 \% ; n=8001)$, regular breakfast patterns $(86.3 \% ; n=4022)$, and had a higher percentage of parents with high education level (62.3\%; $n=1567$ ) (Table 3). Boys were over-represented among unhealthy eaters $(61.5 \% ; n=798)$, as were girls among healthy eaters $(59.5 \% ; n=2775)$ (Table 3$)$. Irregular breakfast patterns were more common in girls $(56.3 \%$; $n=1130$ ) than in boys. Moreover, there was a higher proportion of older children with irregular meal patterns $(14.3 \% ; n=367)$ and irregular breakfast patterns $(14.5 \% ; n=291)$ (Tables 4 and 5).

The correspondence analysis summarized the associations of children's characteristics with eating habits, meal patterns, and breakfast patterns, and confirmed the results presented in Tables 3-5. The resultant graphic representation of the combined results shows a clustering of irregular meal patterns, irregular breakfast patterns, foreign background, and older children (Fig. 2). Unhealthy eaters were more associated with male gender and younger age. Healthy eaters were clustered with regular meal pattern and regular breakfast pattern and were associated with female gender.

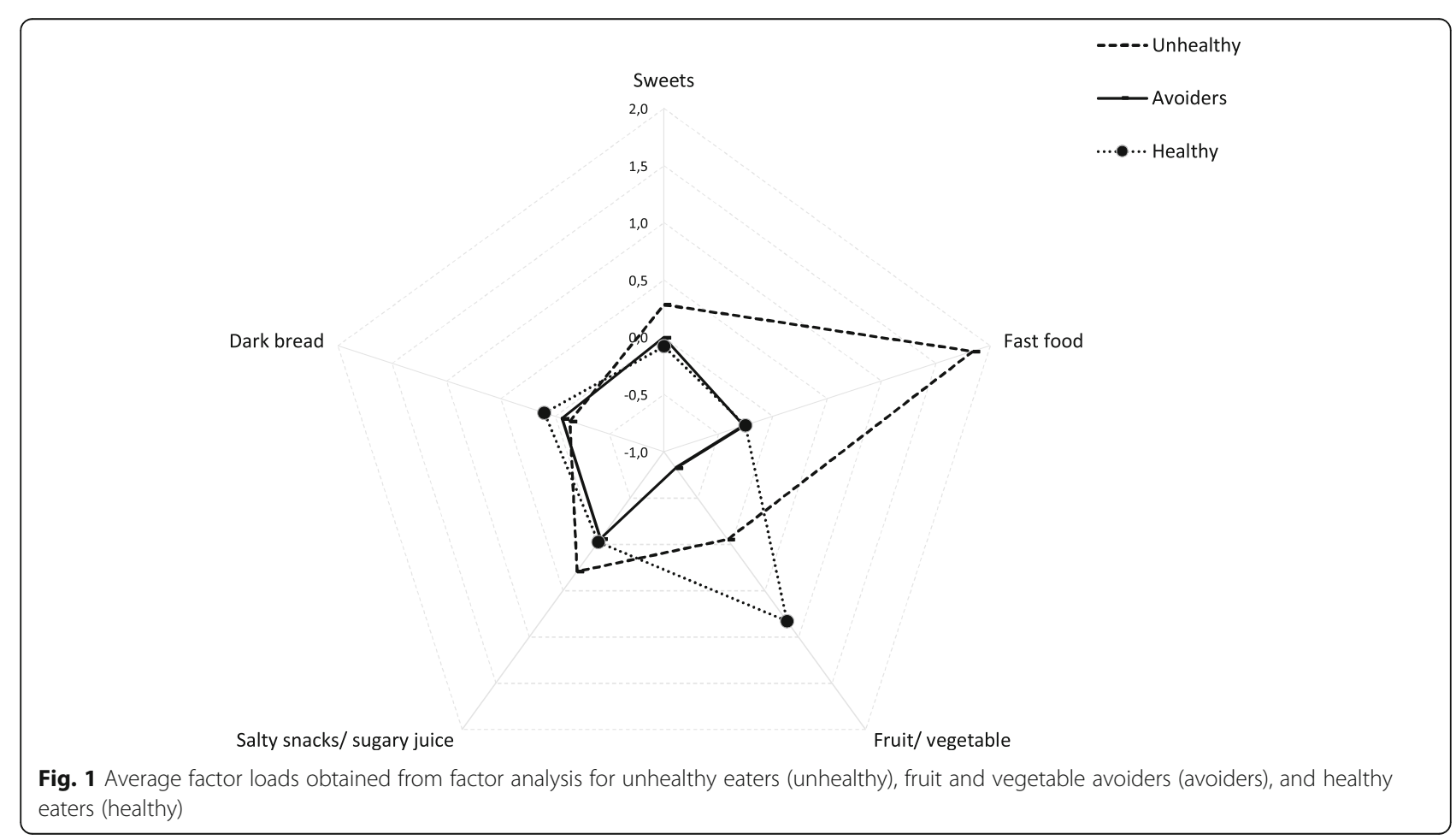


Table 2 Consumption of the 14 food items included in the food frequency questionnaire among unhealthy eaters, fruit and vegetable avoiders, and healthy eaters

\begin{tabular}{|c|c|c|c|c|c|c|c|c|c|c|}
\hline & & \multicolumn{2}{|c|}{ Unhealthy eaters } & \multicolumn{2}{|c|}{ Fruit and vegetable avoiders } & \multicolumn{2}{|c|}{ Healthy eaters } & \multicolumn{2}{|l|}{ Total } & \multirow{2}{*}{$\begin{array}{l}p- \\
\text { value }^{a}\end{array}$} \\
\hline & & $\mathrm{n}$ & $\%$ & $n$ & $\%$ & $n$ & $\%$ & $n$ & $\%$ & \\
\hline \multirow[t]{3}{*}{ Dark grain bread } & Maximum once a week & 323 & $24.9 \%$ & 1358 & $29.5 \%$ & 528 & $11.3 \%$ & 2209 & $20.9 \%$ & \multirow[t]{3}{*}{$<0.001$} \\
\hline & 2-6 times per week & 621 & $47.8 \%$ & 2451 & $53.2 \%$ & 2237 & $48.0 \%$ & 5309 & $50.2 \%$ & \\
\hline & At least once a day & 354 & $27.3 \%$ & 801 & $17.4 \%$ & 1896 & $40.7 \%$ & 3051 & $28.9 \%$ & \\
\hline \multirow[t]{3}{*}{ Fresh or grated vegetables/ salad } & Maximum once a week & 299 & $23.0 \%$ & 1538 & $33.4 \%$ & 33 & $0.7 \%$ & 1870 & $17.7 \%$ & \multirow[t]{3}{*}{$<0.001$} \\
\hline & 2-6 times per week & 639 & $49.2 \%$ & 2682 & $58.2 \%$ & 1144 & $24.5 \%$ & 4465 & $42.2 \%$ & \\
\hline & At least once a day & 360 & $27.7 \%$ & 390 & $8.5 \%$ & 3484 & $74.7 \%$ & 4234 & $40.1 \%$ & \\
\hline \multirow[t]{3}{*}{ Fruits or berries } & Maximum once a week & 251 & $19.3 \%$ & 1992 & $43.2 \%$ & 72 & $1.5 \%$ & 2315 & $21.9 \%$ & \multirow[t]{3}{*}{$<0.001$} \\
\hline & 2-6 times per week & 685 & $52.8 \%$ & 2475 & $53.7 \%$ & 1641 & $35.2 \%$ & 4801 & $45.4 \%$ & \\
\hline & At least once a day & 362 & $27.9 \%$ & 143 & $3.1 \%$ & 2948 & $63.2 \%$ & 3453 & $32.7 \%$ & \\
\hline \multirow[t]{3}{*}{ Sweet pastry } & Less than once a week & 493 & $38.0 \%$ & 2896 & $62.8 \%$ & 3115 & $66.8 \%$ & 6504 & $61.5 \%$ & \multirow[t]{3}{*}{$<0.001$} \\
\hline & Once a week & 353 & $27.2 \%$ & 1022 & $22.2 \%$ & 1003 & $21.5 \%$ & 2378 & $22.5 \%$ & \\
\hline & more than once a week & 452 & $34.8 \%$ & 692 & $15.0 \%$ & 543 & $11.6 \%$ & 1687 & $16.0 \%$ & \\
\hline \multirow[t]{3}{*}{ Biscuits/ cookies } & Less than once a week & 352 & $27.1 \%$ & 2023 & $43.9 \%$ & 2294 & $49.2 \%$ & 4669 & $44.2 \%$ & \multirow[t]{3}{*}{$<0.001$} \\
\hline & Once a week & 333 & $25.7 \%$ & 1188 & $25.8 \%$ & 1134 & $24.3 \%$ & 2655 & $25.1 \%$ & \\
\hline & more than once a week & 613 & $47.2 \%$ & 1399 & $30.3 \%$ & 1233 & $26.5 \%$ & 3245 & $30.7 \%$ & \\
\hline \multirow[t]{3}{*}{ Ice cream } & Less than once a week & 378 & $29.1 \%$ & 3183 & $69.0 \%$ & 2967 & $63.7 \%$ & 6528 & $61.8 \%$ & \multirow[t]{3}{*}{$<0.001$} \\
\hline & Once a week & 359 & $27.7 \%$ & 908 & $19.7 \%$ & 957 & $20.5 \%$ & 2224 & $21.0 \%$ & \\
\hline & more than once a week & 561 & $43.2 \%$ & 519 & $11.3 \%$ & 737 & $15.8 \%$ & 1817 & $17.2 \%$ & \\
\hline \multirow[t]{3}{*}{ Sugary juice drinks } & Less than once a week & 296 & $22.8 \%$ & 2229 & $48.4 \%$ & 2026 & $43.5 \%$ & 4551 & $43.1 \%$ & \multirow[t]{3}{*}{$<0.001$} \\
\hline & Once a week & 278 & $21.4 \%$ & 1028 & $22.3 \%$ & 1037 & $22.2 \%$ & 2343 & $22.2 \%$ & \\
\hline & more than once a week & 724 & $55.8 \%$ & 1353 & $29.3 \%$ & 1598 & $34.3 \%$ & 3675 & $34.8 \%$ & \\
\hline \multirow[t]{3}{*}{ Pizza } & Not at all & 21 & $1.6 \%$ & 1150 & $24.9 \%$ & 1182 & $25.4 \%$ & 2353 & $22.3 \%$ & \multirow[t]{3}{*}{$<0.001$} \\
\hline & Less than once a week & 320 & $24.7 \%$ & 3165 & $68.7 \%$ & 3225 & $69.2 \%$ & 6710 & $63.5 \%$ & \\
\hline & At least once a week & 957 & $73.7 \%$ & 295 & $6.4 \%$ & 254 & $5.4 \%$ & 1506 & $14.2 \%$ & \\
\hline \multirow[t]{3}{*}{ Hamburger or hot dog } & Not at all & 29 & $2.2 \%$ & 1255 & $27.2 \%$ & 1573 & $33.7 \%$ & 2857 & $27.0 \%$ & $<0.001$ \\
\hline & Less than once a week & 311 & $24.0 \%$ & 3066 & $66.5 \%$ & 2895 & $62.1 \%$ & 6272 & $59.3 \%$ & \\
\hline & At least once a week & 958 & $73.8 \%$ & 289 & $6.3 \%$ & 193 & $4.1 \%$ & 1440 & $13.6 \%$ & \\
\hline Salty & Not at all & 24 & $1.8 \%$ & 448 & $9.7 \%$ & 515 & $11.0 \%$ & 987 & $9.3 \%$ & $<0.001$ \\
\hline & Less than once a week & 167 & $12.9 \%$ & 2114 & $45.9 \%$ & 2219 & $47.6 \%$ & 4500 & $42.6 \%$ & \\
\hline & At least once a week & 1107 & $85.3 \%$ & 2048 & $44.4 \%$ & 1927 & $41.3 \%$ & 5082 & $48.1 \%$ & \\
\hline Milk or soured milk & Less than 4 times a week & 230 & $17.7 \%$ & 858 & $18.6 \%$ & 458 & $9.8 \%$ & 1546 & $14.6 \%$ & $<0.001$ \\
\hline & Almost once a day & 304 & $23.4 \%$ & 1045 & $22.7 \%$ & 689 & $14.8 \%$ & 2038 & $19.3 \%$ & \\
\hline & Several times a day & 763 & $58.8 \%$ & 2706 & $58.7 \%$ & 3514 & $75.4 \%$ & 6983 & $66.1 \%$ & \\
\hline Cooked vegetables & Maximum once a week & 775 & $59.7 \%$ & 3273 & $71.0 \%$ & 2066 & $44.3 \%$ & 6114 & $57.9 \%$ & $<0.001$ \\
\hline & Almost once a day & 396 & $30.5 \%$ & 1207 & $26.2 \%$ & 1855 & $39.8 \%$ & 3458 & $32.7 \%$ & \\
\hline & Several times a day & 127 & $9.8 \%$ & 128 & $2.8 \%$ & 738 & $15.8 \%$ & 993 & $9.4 \%$ & \\
\hline Fresh juice & Less than once a week & 223 & $17.2 \%$ & 1446 & $31.4 \%$ & 888 & $19.1 \%$ & 2557 & $24.2 \%$ & $<0.001$ \\
\hline & $1-4$ times a week & 533 & $41.1 \%$ & 2082 & $45.2 \%$ & 1700 & $36.5 \%$ & 4315 & $40.8 \%$ & \\
\hline & 5-6 times a week or more & 541 & $41.7 \%$ & 1081 & $23.5 \%$ & 2073 & $44.5 \%$ & 3695 & $35.0 \%$ & \\
\hline Soft drink & Less than once a week & 249 & $19.2 \%$ & 2296 & $49.8 \%$ & 2536 & $54.4 \%$ & 5081 & $48.1 \%$ & $<0.001$ \\
\hline & Almost once a week & 804 & $62.0 \%$ & 2161 & $46.9 \%$ & 2000 & $42.9 \%$ & 4965 & $47.0 \%$ & \\
\hline & 5-6 times a week or more & 244 & $18.8 \%$ & 149 & $3.2 \%$ & 123 & $2.6 \%$ & 516 & $4.9 \%$ & \\
\hline
\end{tabular}


Table 3 Meal patterns, breakfast patterns, and socio-demographic characteristics of unhealthy eaters, fruit and vegetable avoiders, and healthy eaters

\begin{tabular}{|c|c|c|c|c|c|c|c|c|}
\hline & & \multicolumn{6}{|c|}{ Eating habits group } & \multirow{3}{*}{$\begin{array}{l}p^{-} \\
\text {value }^{a}\end{array}$} \\
\hline & & \multicolumn{2}{|c|}{ Unhealthy eaters } & \multicolumn{2}{|c|}{ Fruit and vegetable avoiders } & \multicolumn{2}{|c|}{ Healthy eaters } & \\
\hline & & $n$ & $\%$ & $n$ & $\%$ & $n$ & $\%$ & \\
\hline \multirow[t]{2}{*}{ Meal (lunch/ dinner) pattern } & Irregular & 413 & $31.8 \%$ & 1294 & $28.1 \%$ & 861 & $18.5 \%$ & $<0.001$ \\
\hline & Regular & 885 & $68.2 \%$ & 3316 & $71.9 \%$ & 3800 & $81.5 \%$ & \\
\hline \multirow[t]{2}{*}{ Breakfast pattern } & Irregular & 318 & $24.5 \%$ & 1049 & $22.8 \%$ & 639 & $13.7 \%$ & $<0.001$ \\
\hline & Regular & 980 & $75.5 \%$ & 3561 & $77.2 \%$ & 4022 & $86.3 \%$ & \\
\hline \multirow[t]{2}{*}{ Gender } & Girl & 500 & $38.5 \%$ & 2289 & $49.7 \%$ & 2775 & $59.5 \%$ & $<0.001$ \\
\hline & Boy & 798 & $61.5 \%$ & 2321 & $50.3 \%$ & 1886 & $40.5 \%$ & \\
\hline \multirow[t]{3}{*}{ Age } & $<11$ years & 432 & $33.3 \%$ & 1303 & $28.3 \%$ & 1184 & $25.4 \%$ & $<0.001$ \\
\hline & 11 years & 728 & $56.1 \%$ & 2780 & $60.3 \%$ & 2949 & $63.3 \%$ & \\
\hline & $>11$ years & 138 & $10.6 \%$ & 527 & $11.4 \%$ & 528 & $11.3 \%$ & \\
\hline \multirow[t]{3}{*}{ Language spoken at home } & Finnish & 1182 & $91.1 \%$ & 4324 & $93.8 \%$ & 4344 & $93.2 \%$ & $<0.001$ \\
\hline & Swedish & 52 & $4.0 \%$ & 185 & $4.0 \%$ & 208 & $4.5 \%$ & \\
\hline & Others & 64 & $4.9 \%$ & 101 & $2.2 \%$ & 109 & $2.3 \%$ & \\
\hline \multirow[t]{2}{*}{ Parental education level ${ }^{b}$} & Low & 343 & $55.0 \%$ & 1218 & $50.1 \%$ & 948 & $37.7 \%$ & $<0.001$ \\
\hline & High & 281 & $45.0 \%$ & 1215 & $49.9 \%$ & 1567 & $62.3 \%$ & \\
\hline
\end{tabular}

results for Chi-square test

banything until technical high school was categorized as low education level; anything higher was classified as high education level

\section{Discussion}

We identified three groups of children with different eating habits: unhealthy eaters, fruit and vegetable avoiders, and healthy eaters. The meal and breakfast patterns of these groups also differed, as did the socio-demographic characteristics: gender, age and language spoken at home.

All participants were pupils in elementary/primary public schools in Finland, where school lunch is served every school day, free of charge [34]. School lunch provides $20 \%$ of daily energy intake [9], underlining that most of the differences in adolescents' food intake depend on food choices made outside school. Since we were interested in eating habits, we focused on key food items as indicators of healthy or unhealthy eating habits and also in those commonly consumed between meals or as snacks. Children may get snacks from vending machines, school kitchen or bring from home. In several schools in Finland, pupils are able to buy snacks from vending machines or from the school kitchen. Healthiness of these snacks are of concern, since it is difficult to monitor and even more difficult to intervene $[9,35]$.

We identified eating habits using factor analysis and cluster analysis. In total, five distinctive factors were identified: fast food, sweets, salty snacks and sugary juice drinks, dark grain bread, and fruits and vegetables. More generally, these five factors illustrated food items that were correlated with each other. Our results are somewhat similar to dietary patterns that have been described in the Finnish population [5, 12, 36, 37].
In our cohort, $34.7 \%$ of children consumed sugary juice drinks more than once a week, and $4.9 \%$ consumed soft drinks at least 5-6 times per week [11]. A sweet dietary pattern has been recognized in various previous nutrition studies in Finland [38, 39], including a study by Bingham et al., which noted that sweet foods constituted a notable part of the diet of Finnish army recruits, and were typically consumed as snacks between meals, or used to replace meals [39]. Moreover, sugar-sweetened drinks are common sources of sucrose in preschool and school-aged children [11]. Interestingly, $66.1 \%$ of our children consumed milk or sour milk several times a day, $32.4 \%$ reported eating cooked vegetables, most likely potatoes, almost once a day, and $29 \%$ had dark grain bread at least once a day, which illuminate the traditional Finnish dietary pattern [37]. Dark grain bread, especially rye bread is a traditional food item in Finland [37] and seems to be popular across different age groups, with similar patterns reported in young military recruits [39], young children [11], and pregnant women [5]. A dietary pattern with fruits and vegetables was identified in our children as well, with $32.7 \%$ reporting to eat fruits and berries and $40.1 \%$ reporting to eat fresh or grated vegetables at least once a day, which showed lower consumption frequency of vegetables, fruits and berries than recommended [40]. Our study did not provide information on quantity, only frequency of consumption. Previous studies support our findings and have reported similar or even lower portions for daily vegetable, fruit, and berry user among 
Table 4 Meal patterns (lunch and dinner) according to eating habits, breakfast patterns, and socio-demographic characteristics

\begin{tabular}{|c|c|c|c|c|c|c|}
\hline & & \multicolumn{4}{|c|}{ Meal pattern (lunch/dinner) } & \multirow{3}{*}{$\begin{array}{l}\text { p- } \\
\text { value }^{a}\end{array}$} \\
\hline & & \multicolumn{2}{|c|}{ Irregular } & \multicolumn{2}{|c|}{ Regular } & \\
\hline & & $\mathrm{n}$ & $\%$ & $n$ & $\%$ & \\
\hline \multirow[t]{3}{*}{ Eating habits } & Unhealthy & 413 & $16.1 \%$ & 885 & $11.1 \%$ & $<0.001$ \\
\hline & Avoider & 1294 & $50.4 \%$ & 3316 & $41.4 \%$ & \\
\hline & Healthy & 861 & $33.5 \%$ & 3800 & $47.5 \%$ & \\
\hline \multirow[t]{2}{*}{ Breakfast pattern } & Irregular & 913 & $35.6 \%$ & 1093 & $13.7 \%$ & $<0.001$ \\
\hline & Regular & 1655 & $64.4 \%$ & 6908 & $86.3 \%$ & \\
\hline \multirow[t]{2}{*}{ Gender } & Girls & 1390 & $54.1 \%$ & 4174 & $52.2 \%$ & 0.084 \\
\hline & Boys & 1178 & $45.9 \%$ & 3827 & $47.8 \%$ & \\
\hline \multirow[t]{3}{*}{ Age } & $<11$ years & 666 & $25.9 \%$ & 2253 & $28.2 \%$ & $<0.001$ \\
\hline & 11 years & 1535 & $59.8 \%$ & 4922 & $61.5 \%$ & \\
\hline & $>11$ years & 367 & $14.3 \%$ & 826 & $10.3 \%$ & \\
\hline \multirow[t]{3}{*}{ Language spoken at home } & Finnish & 2362 & $92.0 \%$ & 7488 & $93.6 \%$ & $<0.001$ \\
\hline & Swedish & 102 & $4.0 \%$ & 343 & $4.3 \%$ & \\
\hline & Others & 104 & $4.0 \%$ & 170 & $2.1 \%$ & \\
\hline \multirow[t]{2}{*}{ Parental education level ${ }^{b}$} & Low & 613 & $50.2 \%$ & 1896 & $43.6 \%$ & $<0.001$ \\
\hline & High & 607 & $49.8 \%$ & 2456 & $56.4 \%$ & \\
\hline
\end{tabular}

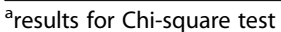

banything until technical high school was categorized as low education level; anything higher was classified as high education level

Table 5 Breakfast patterns according to eating habits, meal patterns, and socio-demographic characteristics

\begin{tabular}{|c|c|c|c|c|c|c|}
\hline & & \multicolumn{4}{|c|}{ Breakfast pattern } & \multirow{3}{*}{$\begin{array}{l}p^{-} \\
\text {value }^{a}\end{array}$} \\
\hline & & \multicolumn{2}{|c|}{ Irregular } & \multicolumn{2}{|c|}{ Regular } & \\
\hline & & $n$ & $\%$ & $n$ & $\%$ & \\
\hline \multirow[t]{3}{*}{ Eating habits } & Unhealthy & 709 & $35.3 \%$ & 3116 & $36.4 \%$ & $<0.001$ \\
\hline & Avoider & 674 & $33.6 \%$ & 3066 & $35.8 \%$ & \\
\hline & Healthy & 623 & $31.1 \%$ & 2381 & $27.8 \%$ & \\
\hline \multirow[t]{2}{*}{ Meal (lunch/ dinner) pattern } & Irregular & 913 & $45.5 \%$ & 1655 & $19.3 \%$ & $<0.001$ \\
\hline & Regular & 1093 & $54.5 \%$ & 6908 & $80.7 \%$ & \\
\hline \multirow[t]{2}{*}{ Gender } & Girls & 1130 & $56.3 \%$ & 4434 & $51.8 \%$ & $<0.001$ \\
\hline & Boys & 876 & $43.7 \%$ & 4129 & $48.2 \%$ & \\
\hline \multirow[t]{3}{*}{ Age } & $<11$ years & 472 & $23.5 \%$ & 2447 & $28.6 \%$ & $<0.001$ \\
\hline & 11 years & 1243 & $62.0 \%$ & 5214 & $60.9 \%$ & \\
\hline & $>11$ years & 291 & $14.5 \%$ & 902 & $10.5 \%$ & \\
\hline \multirow[t]{3}{*}{ Language spoken at home } & Finnish & 1962 & $91.9 \%$ & 8174 & $93.3 \%$ & $<0.001$ \\
\hline & Swedish & 69 & $3.2 \%$ & 404 & $4.6 \%$ & \\
\hline & Others & 105 & $4.9 \%$ & 181 & $2.1 \%$ & \\
\hline \multirow[t]{2}{*}{ Parental education level ${ }^{b}$} & Low & 571 & $57.7 \%$ & 1999 & $42.6 \%$ & $<0.001$ \\
\hline & High & 419 & $42.3 \%$ & 2697 & $57.4 \%$ & \\
\hline
\end{tabular}




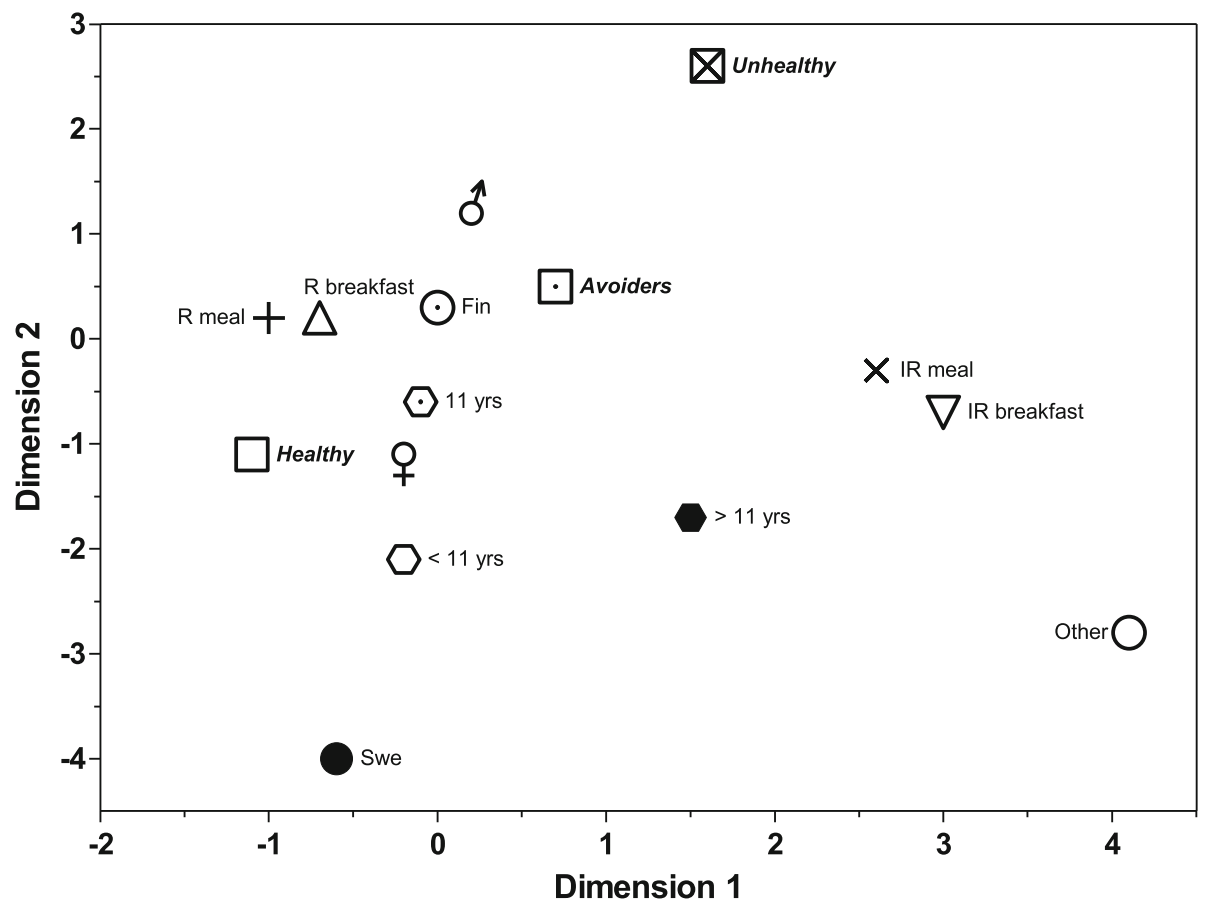

Fig. 2 Map of results of the correspondence analysis. * eating habits are presented with different kind of squares, regular and irregular meal patterns with crosses, regular and irregular breakfast with triangles, languages with circles (Swe - Swedish; Fin - Finnish), age groups with hexagons and gender. R; regular, IR; irregular

Finnish children and adolescents $[9,11,12]$. These foods are typically linked to healthy or health-conscience dietary patterns $[5,37,38,41]$, but less to the traditional Finnish diet [37].

In our study, $44.1 \%$ were healthy eaters and $43.6 \%$ were fruit and vegetable avoiders. The avoiders group ate less sweets and fast foods, but they did not choose fruits or vegetables either. Unhealthy eaters made up the smallest proportion of our cohort (12.3\%). They mostly consumed fast food, sweets, and sugary drinks. Unhealthy eaters were mostly boys and younger children, and their parents had a lower education level compared with the other groups. The foods items characterizing the unhealthy eaters in our study were similar to those found in ISCOLE, a multi-national study [36]. Although it is unclear, the association between unhealthy diet and gender has been reported in several studies around the world, in which boys have consistently been over-represented in groups with unhealthy diet [42, 43]. It has been reported that girls eat more fruits and vegetables than boys [44]. This shows more healthy behaviour among girls, which is expected since it has been suggested that they also have a higher affinity for vegetables and have fewer perceived barriers to their consumption [44, 45]. A previous study pointed out that among male adolescents a healthier diet is associated with less peer pressure, and is positively correlated with adolescents' self-confidence [46]. Thus, programs should work to change the perception of healthy eating so it is also seen as a masculine habit.

Fruit and vegetable avoiders ate the least fruits and vegetables, even lesser than unhealthy eaters. However, avoiders did not eat unhealthy foods, and the majority had both regular meal and regular breakfast consumption, suggesting they are less likely to eat or drink between meals. Nevertheless, several studies have shown that reduced consumption of fruits and vegetables is associated with overweight. One possible explanation for this group is a possible association between avoidance of fruit and vegetable consumption with overweight and under-reporting. Studies have shown under-reporting of food consumption is common in adolescents [47, 48]. The HELENA study reported that obese and overweight adolescents were more likely to under-report food intake, while underweight adolescents were more likely to over-report [47]. Older age is also associated with under-reporting of food and drink intakes among adolescents [48], and in our study a higher proportion of older children were observed both in the fruit and vegetable avoider and healthy eater groups.

Regular meal and breakfast consumption are part of the healthy diet [40], whereas unhealthy behaviours such as skipping breakfast or lunch or consuming high amounts of unhealthy food are associated with the development of non-communicable diseases, especially metabolic syndrome $[4,49,50]$. In this study, we 
evaluated meal patterns as lunch and dinner only, and looked at breakfast patterns separately. We found that most children consumed breakfast, as well as lunch and dinner every school day. In general, children with an irregular meal pattern had also an irregular breakfast pattern, which is considered unhealthy. Moreover, skipping lunch and breakfast increases the chance of an unhealthy diet among adults and adolescents in Nordic countries [19]. Much emphasis is placed on breakfast in school-aged children, as it is associated with the intake of nutrients that are important for young adolescents' health $[51,52]$. Skipping breakfast was more common in girls and older children, which is consistent with previous studies [30, 31, 51, 53]. The consumption of breakfast among women varies between countries, but a lower consumption has been noted among women from the Nordic countries [16]. However, in Finland, the consumption of daily breakfast in adolescents girls increased from 2002 to 2010, while this trend was reversed among boys [16].

We included language spoked at home and parental education level in order to evaluate the socioeconomic status of children. Previous Finnish studies have shown that Swedish-speakers have higher socioeconomic status, while immigrants have lower income than the general Finnish population [23, 24, 54]. The association between low socioeconomic status and unhealthy diet behaviour is well-established [55-58]. In the present study, the association between parental education level and children' eating habits and meal/breakfast patterns was only investigated in a subset of participants. Low parental education level and foreign language was more prominently associated with unhealthy eating habits, irregular meal patterns, and skipping breakfast. Our results are consistent with studies showing that lower socioeconomic status is associated with poor quality of the diet, high consumption of fast foods and sweets, and lower consumption of fruits and vegetables [56, 57]. The DIATROFI study showed that daily consumption of breakfast was associated with a higher socioeconomic status [57]. Students with low socioeconomic status have also been shown to have an increased risk of skipping breakfast, lunch, and dinner [58].

The large, nationwide cohort of children is an ultimate strength of our study. Although the participation rate was low (30\%), the distribution of socio-demographic characteristics (such as BMI, gender, maternal language) in our cohort were similar to Finnish children population [20]. Moreover, this large sample size allowed us to identify three distinctive eating habits. Importantly, we were able to characterize a small group of children with unhealthy eating habits. Our findings are consistent with others previous studies, although the FFQ had a short number of food items, as usually is used in this type of school studies $[18,28,29]$ since there are limitations in carrying out long questionnaires with children and adolescents $[25,26]$. We lacked information on the whole diet e.g., food consumption during main meals and meal consumption during weekends. In addition, we were not able to calculate total energy intake since FFQ included only a limited number of food items. Moreover, the FFQ has not been validated. We could assume some inaccuracy in food intake, since information was self-reported by 9-14-year-old children, but it was out of the scope of this study. However, an earlier study showed reliable results in similar FFQ among 11-year old and older children [29]. Furthermore, a qualitative evaluation of the questionnaire in this age group was carried out at the beginning of the study to check the children's cognitive maturity [20]. Another weakness was that parental education level was available for $57 \%$ of children, but despite this, our results were similar with those of other studies.

\section{Conclusion}

In conclusion, we identified three groups of children with different eating habits: unhealthy eaters, fruit and vegetable avoiders, and healthy eaters. A low percentage of our children were unhealthy eaters, and a high proportion of these were boys. In addition, association between unhealthy eating habits and irregular meal/ breakfast patterns were observed. Special attention should be paid to avoider eaters since they have a low consumption of fruits, berries and vegetables, which is typically associated with increased risk of obesity and common chronic diseases. Most of the children had regular meal and regular breakfast patterns. In general, those with irregular meal patterns tended to have irregular breakfast patterns as well, although skipping breakfast was more common among girls and older children. This is the first study to evaluate eating habits and their association with meal patterns and breakfast consumption among young children in Finland. Our results increase understanding on unhealthy eating habits in children and provide further arguments for public health interventions in order to improve healthy eating behaviours.

\section{Abbrevations}

FFQ: Food frequency questionnaire.; Fin-HIT: The Finnish Health in Teens.; HBSC: Health Behaviour in School-Aged Children.; KMO: The Kaiser-MeyerOlklin

\section{Acknowledgments}

The group thanks the adolescents and parents who took part in the Finnish Health in Teens cohort (Fin-HIT) study, all teachers and principals of the schools, fieldworkers and coordinators who took part in cohort enrolment.

\section{Funding}

Academy of Finland [grant number 250704]; Life and Health Medical Fund [grant number 1-23-28]; The Swedish Cultural Foundation in Finland [grant number 15/0897]; Signe and Ane Gyllenberg Foundation [grant number 371977-43]; and Yrjö Jahnsson Foundation [grant number 11486]. The funders 
had no role in study design, data collection and analysis, decision to publish, or preparation of the manuscript.

\section{Availability of data and materials}

The data sets analyzed in this study were obtained from Finnish Health in Teens (Fin-HIT) study. Data are available and can be obtained from Folkäalsan Research Center on a formal and reasonable request. Contact the corresponding author.

\section{Authors' contributions}

All authors designed the study. RAOF, JV and HV conducted literature searches and provided summaries on previous studies. RAOF and JV conducted the statistical analysis. EW, TBR and ER made substantial contributions to conception and design of the study. EW is responsible for acquisition of data. All authors have interpreted the results. RAOF wrote the first draft of the manuscript and all authors have critically revised, and approved the final version of manuscript.

\section{Ethics approval and consent to participate}

The Fin-HIT study protocol was approved by the Coordinating Ethics Committee of the Helsinki and Uusimaa Hospital District (decision number 169/ 13/03/00/10). Informed written consent was obtained from all children and from one legally responsible adult per each child (i.e. parent or legal guardian) according to the Helsinki Declaration.

\section{Consent for publication}

Not applicable.

\section{Competing interests}

The authors declare that they have no competing interests.

\section{Publisher's Note}

Springer Nature remains neutral with regard to jurisdictional claims in published maps and institutional affiliations.

\section{Author details}

'Folkhälsan Research Center, Biomedicum 1 Helsinki, PB 63 (Haartmansgatan 8), 00014 University of Helsinki, Helsinki, Finland. ${ }^{2}$ Faculty of Medicine, University of Helsinki, Helsinki, Finland. ${ }^{3}$ Department of Food and Environmental Sciences, University of Helsinki, Helsinki, Finland. ${ }^{4}$ Department of Public Health, University of Helsinki, Helsinki, Finland. ${ }^{5}$ Department of Research, Cancer Registry of Norway, Oslo, Norway. ${ }^{6}$ Department of Medical Epidemiology and Biostatistics, Karolinska Institutet, Stockholm, Sweden. ${ }^{7}$ Department of Community Medicine, Faculty of Health Sciences, University of Tromsø, The Arctic University of Norway, Tromsø, Norway.

Received: 15 March 2018 Accepted: 27 February 2019 Published online: 15 March 2019

\section{References}

1. Sofi F, Abbate R, Gensini GF, Casini A. Accruing evidence on benefits of adherence to the Mediterranean diet on health : an updated systematic review and meta-analysis 1, 2. Am J Clin Nutr. 2010;92:1189-96.

2. Winkvist A, Hultén B, Kim J-L, Johansson I, Torén K, Brisman J, et al. Dietary intake, leisure time activities and obesity among adolescents in Western Sweden: a cross-sectional study. Nutr J [Internet]. Nutr J; 2015;15:41. Available from: http://nutritionj.biomedcentral.com/articles/10.1186/s12937016-0160-2.

3. Ng M, Fleming T, Robinson M, Thomson B, Graetz N, Margono C, et al, Global, regional, and national prevalence of overweight and obesity in children and adults during 1980-2013: a systematic analysis for the global burden of disease study 2013. Lancet. 2014;384:766-81.

4. Wennberg $M$, Gustafsson $P E$, Wennberg $P$, Hammarström A. Irregular eating of meals in adolescence and the metabolic syndrome in adulthood: results from a 27-year prospective cohort. Public Health Nutr [Internet]. 2016;19: 667-73 Available from: http://www.journals.cambridge.org/abstract_ S1368980015001445

5. Arkkola T, Uusitalo U, Kronberg-Kippilä C, Männistö S, Virtanen M, Kenward MG, Veijola R, Knip M, Ovaskainen ML VS. Seven distinct dietary patterns identified among pregnant Finnish women--associations with nutrient intake and sociodemographic factors. Public Heal Nutr [Internet]. Cambridge
University Press; 2008 [cited 2017 May 18];11:176-82. Available from: http:// researchonline.Ishtm.ac.uk/7958/.

6. Green R, Sutherland J, Dangour AD, Shankar B, Webb P. Global dietary quality, undernutrition and non-communicable disease: a longitudinal modelling study. BMJ Open [Internet]. 2016;6:e009331. Available from: https://bmjopen. bmj.com/content/6/1/e009331.

7. Raulio S, Roos E, Prättälä R. School and workplace meals promote healthy food habits. Public Health Nutr. 2010;13:987-92.

8. Fismen A-S, Smith O, Torsheim T, Samdal O. A school based study of time trends in food habits and their relation to socio-economic status among Norwegian adolescents, 2001-2009. Int J Behav Nutr Phys Act [Internet]. 2014;11:115 Available from: https://www.ncbi.nlm.nih.gov/pmc/articles/ PMC4177592/pdf/12966_2014_Article_115.pdf.

9. Hoppu U, Lehtisalo J, Tapanainen H, Pietinen P. Dietary habits and nutrient intake of Finnish adolescents. Public Health Nutr. 2010;13:965-72.

10. Kyttälä P, Erkkola M, Kronberg-Kippilä C, Tapanainen H, Veijola R, Simell O, et al. Food consumption and nutrient intake in Finnish 1-6-year-old children. Public Health Nutr [Internet]. 2010;13:947-56 Available from: http://www. journals.cambridge.org/abstract_S136898001000114X.

11. Eloranta a M, Lindi V, Schwab U, Kiiskinen S, Kalinkin M, Lakka HM, et al. Dietary factors and their associations with socioeconomic background in Finnish girls and boys 6-8 years of age: the PANIC Study. Eur J Clin Nutr [Internet]. Nature Publishing Group; 2011;65:12111218. Available from: http://www.ncbi.nlm.nih.gov/pubmed/21697818.

12. Bingham CML, Jallinoja P, Lahti-Koski M, Absetz P, Paturi M, Pihlajamäki H, et al. Quality of diet and food choices of Finnish young men: a sociodemographic and health behaviour approach. Public health Nutr [internet]. 2010;13:980-6 Available from: http://www.ncbi.nlm.nih.gov/ pubmed/20513269.

13. Lehto R, Ray C, Lahti-Koski M, Roos E. Meal pattern and BMI in 9-11year-old children in Finland. Public Health Nutr [Internet]. 2011;14:124550 Available from: http://www.journals.cambridge.org/abstract_ S1368980010003034.

14. Tilles-Tirkkonen T, Suominen S, Liukkonen J, Poutanen K, Karhunen L. Determinants of a regular intake of a nutritionally balanced school lunch among 10-17-year-old schoolchildren with special reference to sense of coherence. J Hum Nutr Diet [Internet]. 2015 [cited 2017 May 18];28:56-63. Available from: http://doi.wiley.com/10.1111/jhn.12221.

15. Roos E, Prättälä R. Meal pattern and nutrient intake among adult Finns. Appetite [Internet]. 1997;29:11-24 Available from: http://linkinghub.elsevier. com/retrieve/pii/S0195666396900951.

16. Lazzeri G, Ahluwalia N, Niclasen B, Pammolli A, Vereecken C, Rasmussen M, et al. Trends from 2002 to 2010 in daily breakfast consumption and its socio-demographic correlates in adolescents across 31 countries participating in the HBSC study. PLoS One. 2016;11:1-13.

17. U.S. Department of Agriculture, U.S. Department of Health and Human Services. Dietary Guidelines for Americans. 7Th Ed. Washington, DC: U.S; 2010. p. 2010

18. Fismen AS, Smith ORF, Torsheim $T$, Rasmussen M, Pagh TP, Augustine $L$, et al. Trends in food habits and their relation to socioeconomic status among nordic adolescents 2001/2002-2009/2010. PLoS One. 2016;11:1-15.

19. Holm L, Lund TB, Niva M. Eating practices and diet quality: a population study of four Nordic countries. Eur J Clin Nutr. 2015;69:791-8.

20. Figueiredo RAO, Simola-Ström S, Rounge TB, Viljakainen H, Eriksson JG, Roos E, et al. Cohort profile: the Finnish health in teens (fin-HIT) study: a population-based study. Int J Epidemiol. 2018.

21. Pudaric S, Sundquist J, Johansson S-E. Country of birth, instrumental activities of daily living, self-rated health and mortality: a Swedish population-based survey of people aged 55-74. Soc Sci Med. 2003;56: 2493-503.

22. Álvarez-Gálvez J, Jaime-Castillo AM. The impact of social expenditure on health inequalities in Europe. Soc Sci med [internet]. Elsevier. 2018;200:9-18 Available from: http://linkinghub.elsevier.com/retrieve/pii/ S0277953618300066

23. Volanen S, Suominen S, Lahelma E, Koskenvuo M, Silventoinen K. Sense of coherence and its determinants: a comparative study of the Finnishspeaking majority and the Swedish-speaking minority in Finland. Scand J Public Health. 2006;34:515-25.

24. Paljärvi T, Suominen $\mathrm{S}$, Koskenvuo M, Winter T, Kauhanen J. The differences in drinking patterns between Finnish-speaking majority and Swedishspeaking minority in Finland. Eur J Pub Health. 2009;19:278-84. 
25. Magarey A, Watson J, Golley RK, Burrows T, Sutherland R, Mcnaughton SA, et al. Assessing dietary intake in children and adolescents: considerations and recommendations for obesity research. Int J Pediatr Obes. 2011;6:2-11.

26. Pérez-Rodrigo C, Escauriaza BA, Escauriaza JA, Allúe IP. Dietary assessment in children and adolescents: issues and recommendations. Nutr Hosp. 2015;31: 76-83.

27. WHO. Health Behaviour in School-Aged Children (HBSC) Study Protocol: Background, Methodology and Mandatory Items for the 2009/ 10 Survey. sugary soft drinks; 2009.

28. Gabhainn SN, Nolan G, Kelleher C, Friel S. Dieting patterns and related lifestyles of school-aged children in the Republic of Ireland. Public Health Nutr. 2002;5:457-62.

29. Vereecken CA, Maes L. A Belgian study on the reliability and relative validity of the Health Behaviour in School-Aged Children food-frequency questionnaire. Public Health Nutr [Internet]. 2003;6:581-8 Available from: http://www.journals.cambridge.org/abstract_S1368980003000788.

30. Keski-Rahkonen A, Kaprio J, Rissanen A, Virkkunen M, Rose RJ. Breakfast skipping and health-compromising behaviors in adolescents and adults. Eur J Clin Nutr [Internet]. 2003;57:842-53 Available from: http://www.nature. com/doifinder/10.1038/sj.ejcn.1601618.

31. Vereecken C, Dupuy M, Rasmussen M, Kelly C, Nansel TR. Sabbah H Al, et al. breakfast consumption and its socio-demographic and lifestyle correlates in schoolchildren in 41 countries participating in the HBSC study. Int J Public Heal. 2012;54:1-15.

32. Hair JF, Black WC, Babin BJ, Anderson RE. Multivariate Data Analysis. 7th ed. Prentice Hall: Upper Saddle River; 2010.

33. Greenacre M, Blasius J. Multiple correspondence analysis and related methods. London: Chapman \& Hall; 2006.

34. Sarlio-Lähteenkorva S, Manninen M. School meals and nutrition education in Finland. Nutr Bull. 2010;35:172-4.

35. Tilles-Tirkkonen T, Pentikäinen S, Lappi J, Karhunen L, Poutanen K, Mykkänen $H$. The quality of school lunch consumed reflects overall eating patterns in 11-16-year-old schoolchildren in Finland. Public health Nutr [internet]. 2011; 14:2092-8 Available from: http://www.ncbi.nlm.nih.gov/pubmed/21752309.

36. Mikkilä V, Vepsäläinen H, Saloheimo T, Gonzalez SA, Meisel JD, Hu G, et al. An international comparison of dietary patterns in 9-11-year-old children. Int J Obes Suppl [Internet]. 2015;5:S17-21 Available from: http://www. nature.com/doifinder/10.1038/ijosup.2015.14

37. Mikkilä V, Räsänen L, Raitakari O, Pietinen P, Viikari J. Consistent dietary patterns identified from childhood to adulthood: the cardiovascular risk in young Finns study. Br J Nutr. 2005;93:923-31.

38. Nissinen K, Mikkilä V, Männistö S, Lahti-Koski M, Räsänen L, Viikari J, et al. Sweets and sugar-sweetened soft drink intake in childhood in relation to adult BMI and overweight. The Cardiovascular Risk in Young Finns Study. Public Health Nutr [Internet]. 2009;12:2018 Available from: http://www. journals.cambridge.org/abstract_S1368980009005849.

39. Bingham CML, Lahti-Koski M, Absetz P, Puukka P, Kinnunen M, Pihlajamäki $H$, et al. Food choices and health during military service: increases in sugarand fibre-containing foods and changes in anthropometric and clinical risk factors. Public Health Nutr [Internet]. 2012;15:1248-55 Available from: http:// www.journals.cambridge.org/abstract S1368980011003351.

40. NCM -Nordic Council of Ministers. Nordic Nutrition Recommendations 2012. Copenhagen; 2012.

41. Hauta-alus HH, Holmlund-Suila EM, Hannu RJ, Enlund-Cerullo M, Rosendahl J, Valkama SM, et al. Season, dietary factors, and physical activity modify 25hydroxyvitamin D concentration during pregnancy. Eur J Nutr. 2017.

42. Conner TS, Thompson LM, Knight RL, Flett JAM, Richardson AC, Brookie KL. The role of personality traits in young adult fruit and vegetable consumption. Front Psychol. 2017:8:1-11.

43. Olson JS, Hummer RA, Harris KM. Gender and Health Behavior Clustering among U.S. Young Adults. Biodemography Soc Biol [Internet]. 2017;63:3-20 Available from: https://www.ncbi.nlm.nih.gov/pmc/articles/PMC5351770/ pdf/nihms836900.pdf.

44. Lehto E, Ray C, Haukkala A, Yngve A, Thorsdottir I, Roos E. Do descriptive norms related to parents and friends predict fruit and vegetable intake similarly among 11-year-old girls and boys? Br J Nutr. 2016;115:168-75.

45. Lehto E, Ray C, Haukkala A, Yngve A, Thorsdottir I, Roos E. Predicting gender differences in liking for vegetables and preference for a variety of vegetables among 11-year-old children. Appetite Elsevier Ltd. 2015;95:285-92.

46. Fitzgerald A, Heary C, Kelly C, Nixon E, Shevlin M. Self-efficacy for healthy eating and peer support for unhealthy eating are associated with adolescents' food intake patterns. Appetite [Internet]. 2013;63:48-58 Available from: https://doi.org/10.1016/j.appet.2012.12.011.

47. Bel-Serrat S, Julián-Almárcegui C, González-Gross M, Mouratidou T, Börnhorst C, Grammatikaki E, et al. Correlates of dietary energy misreporting among European adolescents: the healthy lifestyle in Europe by nutrition in adolescence (HELENA) study. Br J Nutr [Internet]. 2016;115:1439-52 Available from: http://www.journals.cambridge.org/abstract_ S0007114516000283.

48. Barbara M, Livingstone E, Black AE. Markers of the Validity of Reported Energy Intake. J Nutr. 2003;133(Suppl 1).

49. Sjöberg A, Hallberg L, Höglund D, Hulthén L. Meal pattern, food choice, nutrient intake and lifestyle factors in the Göteborg adolescence study. Eur J Clin Nutr [Internet]. 2003;57:1569-78 Available from: http://www.nature com/doifinder/10.1038/sj.ejcn.1601726.

50. Wijnhoven TM, van Raaij JM, Yngve A, Sjöberg A, Kunešová M, Duleva $V$, et al. WHO European childhood obesity surveillance initiative: health-risk behaviours on nutrition and physical activity in 6-9-year-old schoolchildren. Public Health Nutr [Internet]. 2015;18:3108-24 Available from: http://www. journals.cambridge.org/abstract_S1368980015001937.

51. Fayet-Moore F, Kim J, Sritharan N, Petocz P. Impact of breakfast skipping and breakfast choice on the nutrient intake and body mass index of Australian children. Nutrients. 2016:8:1-12.

52. Mullan B, Wong C, Kothe E, O'Moore K, Pickles K, Sainsbury K. An examination of the demographic predictors of adolescent breakfast consumption, content, and context. BMC Public Health [Internet]. BMC Public Health; 2014;14:264. Available from: http://bmcpublichealth. biomedcentral.com/articles/10.1186/1471-2458-14-264.

53. Lazzeri G, Pammolli A, Azzolini E, Simi R, Meoni V, de Wet DR, et al. Association between fruits and vegetables intake and frequency of breakfast and snacks consumption: a cross-sectional study. Nutr J [Internet]. Nutr J; 2013;12:123. Available from: http://nutritionj.biomedcentral.com/ articles/10.1186/1475-2891-12-123.

54. Idehen EE, Koponen P, Härkänen T, Kangasniemi M, Pietilä A, Korhonen T. Disparities in cervical screening participation : a comparison of Russian , Somali and Kurdish immigrants with the general Finnish population. Int J Equity Health. 2018;17:1-9.

55. Kourlaba G, Panagiotakos DB, Mihas K, Alevizos A, Marayiannis K, Mariolis A, et al. Dietary patterns in relation to socio-economic and lifestyle characteristics among Greek adolescents: a multivariate analysis. Public Health Nutr [Internet]. 2009;12:1366 Available from: http://www.journals. cambridge.org/abstract_S1368980008004060.

56. Drouillet-Pinard P, Dubuisson C, Bordes I, Margaritis I, Lioret S, Volatier J-L. Socio-economic disparities in the diet of French children and adolescents: a multidimensional issue. Public Health Nutr [Internet]. 2017;20:870-82 Available from: https://www.cambridge.org/core/product/identifier/ S1368980016002895/type/journal_article.

57. Yannakoulia M, Lykou A, Kastorini CM, Saranti Papasaranti E, Petralias A, Veloudaki A, et al. Socio-economic and lifestyle parameters associated with diet quality of children and adolescents using classification and regression tree analysis: the DIATROFI study. Public Health Nutr [Internet]. 2016;19:33947 Available from: http://www.journals.cambridge.org/abstract_ S136898001500110X.

58. Hong S, Bae HC, Kim HS, Park EC. Variation in meal-skipping rates of Korean adolescents according to socio-economic status: results of the Korea youth risk behavior web-based survey. J Prev Med Public Heal. 2014;47:158-68.

\section{Ready to submit your research? Choose BMC and benefit from:}

- fast, convenient online submission

- thorough peer review by experienced researchers in your field

- rapid publication on acceptance

- support for research data, including large and complex data types

- gold Open Access which fosters wider collaboration and increased citations

- maximum visibility for your research: over $100 \mathrm{M}$ website views per year

At $\mathrm{BMC}$, research is always in progress.

Learn more biomedcentral.com/submission 